\title{
Prise en charge des troubles de la reproduction de la femme blessée médullaire
}

\author{
J.J. LABAT ${ }^{1}$, B. PERROUIN-VERBE ${ }^{2}$ \\ 1Clinique Urologique, Hôtel Dieu, CHU Nantes ; \\ 2 Service de Rééducation Fonctionnelle Polyvalente, Hôpital Saint Jacques, CHU Nantes.
}

\section{RESUME}

La grossesse de la femme paraplégique est d'un pronostic favorable sous la condition d'un suivi spécialisé. Les complications sont dominées par la fréquence des infections urinaires, le risque de thrombophlébites, la survenue d'escarres. La gravité est liée au possible déclenchement d'un syndrome d'hyperréflexie autonome dans les lésions supérieures à T6, syndrome qui doit être prévenu par la mise en place d'une analgésie péridurale au début du travail. L'accouchement doit s'envisager par voie basse, parfois aidé sur le plan instrumental (forceps). Les indications de césariennes ne doivent être qu'obstétricales ou lors d'un syndrome d'HRA non jugulable. Le risque d'accouchement prématuré apparaît plus important dans cette population, imposant une surveillance drastique lors du $3^{\circ}$ trimestre.

Mots clés : Paraplégie, blessé médullaire, procréation, conception, grossesse, reproduction, accouchement

\section{INTRODUCTION}

La présence d'une lésion médullaire chez une femme ne doit en aucun cas être considérée comme une contre-indication à une grossesse, mais comme un challenge entre la patiente et ses praticiens. Depuis les premiers rapports de Robertson et Guttman [8], la plupart des écrits font état de courtes séries ou de revues de la littérature. Approximativement 50\% des lésions médullaires surviennent entre 15 et 25 ans, le nombre de femmes se situant autour de $15-20 \%$. Ceci justifie pleinement le désir de pro- création de cette population, les programmes de rééeducation-réadaptation les autorisant à une vie active et autonome. La meilleure connaissance physiopathologique, notamment des dysfonctionnements végétatifs, la prise en charge et le suivi dans des unités spécifiques a par ailleurs notablement diminué la morbidité et la mortalité de cette population, dont l'espérance de vie a considérablement augmentée durant les deux dernières décennies [13]. L'ensemble de ces éléments font que, si le taux de grossesse reste encore faible dans cette population, il ne peut que s'accroître dans l'avenir.

\section{SÉRIES PUBLIÉES : PETITS ÉCHANTILLONS}

L 'étude de N. Westgren [11] sur la population suédoise recense 29 femmes (dont 9 femmes tétraplégiques) sur 11 années ayant eu un au moins un enfant et un total de 49 grossesses, soit un taux moyen de naissance par an à un dixième du taux attendu de la population générale. Charlifue [3] dans son étude de 1992 sur la sexualité et la procréation de 231 femmes blessées médullaires âgées de 18 à 45 ans, rapporte un taux de grossesse post-traumatique de 0,34 /personne alors que le taux pré-traumatique est de 1,34/personne.

\section{DIFFICULTÉS DE CONCEPTION}

Cette diminution du taux de conception chez la femme blessé médullaire n'est pas contrairement à l'homme paraplégique, lié à un effet délétère de la lésion neurologique

\section{Correspondance :}

Jean-Jacques Labat, Clinique Urologique, Hôtel Dieu, CHU Nantes, 44035 Nantes Cedex

Communication à la Session SALF-AFU des $6^{\circ}$ Journées de la Fédération Française d'Étude de la Reproduction, Lyon, 5-7 septembre 2001 
elle-même sur la fertilité. La femme paraplégique peut avoir des rapports sexuels et en dehors de la phase d'aménorrhée post-traumatique qui survient dans $60 \%$ d'entreelles pendant 5 mois en moyenne, elle garde une fertilité normale.

La difficulté à assumer à la fois la paraplégie et la situation de mère lors de la grossesse, de l' accouchement et pendant toute la phase éducative participe à ce faible taux. En effet un tiers des femmes de l'étude précédente (Charlifue, [3]), déclarent ne plus vouloir d'enfants, $80 \%$ d'entre elles incriminant le traumatisme, les difficultés physiques rendant difficiles la prise en charge d'enfants. De même des différences significatives apparaissent lorsque l'on établit des sous-groupes en fonction des niveaux neurologiques, les femmes tétraplégiques présentant le taux le plus bas de grossesse. Cette même enquête souligne combien les femmes blessées médullaires souffrent d'un manque d'information concernant leur capacité de sexualité et de procréation, ce qui doit nous encourager à inclure dans nos programmes des séances d'information sur ce sujet.

Le concept d'enfants de parents paraplégiques et/ou de parents paraplégiques est malheureusement trop souvent répandu. Une étude de Westgren de 1994 [12] portant sur les familles de 26 blessées médullaires objective une qualité de vie familiale incontestable (la question devrait-elle se poser ?), la plupart des enfants de cette enquête considérant que la déficience présentée par leur mère n'entraîne en rien la moindre restriction dans leurs activités et échanges sociaux.

\section{DONNÉES PHYSIOPATHOLOGIQUES}

L'état sous lésionnel d'une femme blessée médullaire complète (Frankel A) est caractérisé par l'anesthésie sous lésionnelle, l'existence d'un déficit moteur et la libération des automatismes médullaires dans les atteintes centrales. Lors de lésion spinale de niveau supérieur à T10, il existe une anesthésie des organes génitaux internes et plus particulièrement de l'utérus, ne permettant pas la sensation objective des contractions à l'origine de la notion classique de travail pouvant passer inaperçu, mais des perceptions indirectes sont possibles. Lors de lésions entre T10 et T12 les sensations sont inconstantes, et les femmes présentant une lésion inférieurs à $\mathrm{T} 12$ auront la perception des contractions utérines, de l'effacement du col et de sa dilatation. Lors de lésions complètes à partir de S2 (cônes terminaux ou syndromes de la queue de cheval), il existe une anesthésie des organes génitaux externes et du périnée ne permettant pas de sentir le passage de la filière vagino-périnéale. Quand la lésion est supérieure à T5-T6, le risque d'hyperréflexie autonome est important.

\section{GROSSESSE, ACCOUCHEMENT ET HYPERRÉFLEXIE AUTONOME}

Le syndrome d'hyperréflexie autonome (HRA) a été décrit au début du siècle (1917) par Head et Riddoch [6], ce syndrome est retrouvé dans $85 \%$ des lésions supérieures ou égales à $\mathrm{T} 6$, mais a été rapporté chez une patiente de niveau T10 [1]. Cet orage végétatif lié à la déafférentation du sympathique de ses centres régulateurs supérieurs peut être provoqué par n'importe quel stimulus nociceptif sous lésionnel et plus particulièrement de la sphère sacrée, les causes les plus fréquentes étant la distension vésicale et dans notre contexte les contractions utérines. Les tableaux cliniques de ce syndrome d'HRA sont variés d'un point de vue qualitatif et quantitatif. Les signes cliniques subjectifs associent de façon variable une sensation de nausée, de malaise général, des céphalées pulsatiles parfois extrêmement violentes. D'un point de vue objectif l'hyperactivité sympathique se manifeste par une hypersudation sus-lésionnelle, une érection pilomotrice, des tremblements, une congestion nasale, des phénomènes de flush de la face, une augmentation de la spasticité. La gravité de ce syndrome est liée à l'existence d'une hypertension artérielle, conséquence de la vasoconstriction sous lésionnelle et notamment du territoire splanchnique, pouvant être à l'origine d'hémorragie sous arachnoïdienne, d'accident vasculaire cérébral et de décès. La caractéristique clinique de ce syndrome est d'associer à cette HTA, une bradycardie sinusale (réponse vagale). Ce syndrome ne doit pas être confondu avec un tableau de prééclampsie, ne serait ce que par l'absence d'une protéinurie massive. Le possible déclenchement de ce syndrome d'HRA dans les lésions hautes notamment lors du début du travail et de l'accouchement imposent la prise en charge de ces femmes par des équipes obstétricales rodées conjointement à des médecins paraplégistes. La prévention passe par la limitation des afférences nociceptives locales, par le contrôle de la vacuité vésicale et rectale, par la mise en place d'une anesthésie péridurale continue dès le début du travail (consensus dans la littérature), mais aussi par l'utilisation de gels anesthésiques lors de réalisation d'examens gynécologiques tout au long du suivi, lors de changement de cathéter urinaire, et par la vérification de la qualité de la vidange vésicale tout au long de la grossesse. Eviter toute distension rectale et vésicale

Des cas d'HRA ont été rapportés dans le pré-partum, l'intra partum, le post-partum. L'étude de Westgren [11] en retrouve 2 cas lors de l'analyse des dossiers de ses 29 femmes, mais l'interrogatoire de ces mêmes femmes permet de constater qu'ils sont présents chez $75 \%$ d'entre elles, ceci mettant largement en évidence la méconnaissance de ce syndrome dans les équipes obstétricales. L. L. Cross [4] rapporte une incidence de $56 \%$ dans les lésions $>$ T6. 


\section{LES COMPLICATIONS DE LA GROSSESSE}

\section{Infection urinaire}

Les infections urinaires sont fréquentes chez les blessés médullaires. L'étude de Whiteneck [13] objective une incidence annuelle de $20 \%$ d'infections urinaires dans une étude rétrospective sur 20 ans de 834 blessés médullaires, cette complication étant la deuxième cause de réhospitalisation de ces patients. La présence d'une bactériurie dans la population de femmes enceintes dites saines expose à un risque de $65 \%$ de pyelonéphrites. Chez la femme blessée médullaire, le dysfonctionnement vésico-sphinctérien, quel que soit le mode mictionnel pratiqué, est à l'origine d'une grande fréquence des bactériuries asymptomatiques. Ce risque de bactériurie asymptomatique est de $100 \%$ chez les femmes en sonde à demeure, d'environ $70 \%$ chez les femmes pratiquant l'autosondage. Si la conférence de consensus de 1992 [7] s'accorde pour ne pas traiter les bactériuries asymptomatiques chez les blessés médullaires, cette abstention n'est de règle qu'en l'absence de facteurs de risque telle l'existence d'une obstruction, de hautes pressions intra-vésicales... La grossesse constitue pour les acteurs de cette conférence un facteur de risque, toute bactériurie asymptomatique de la femme paraplégique enceinte doit donc être traitée. Rappelons à cet égard que les critères cytobactériologiques de Kass ont été revus à la baisse dans cette population de blessés médullaires, lors de cette conférence de consensus. S'appuyant sur des études précises, comparant des prélèvements appariés par aspiration suprapubienne et des prélèvements soit de milieu de cathéter chez les patients en autosondage, soit obtenus chez des patients en drainage continu, la conférence de consensus a redéfini ce qu'il convenait d'appeler une bactériurie asymptomatique chez ces patients spinalisés : une bactériurie supérieure à $10^{2} / \mathrm{ml}$ chez les patients en autosondage, et la présence d'une bactériurie à n'importe quelle concentration chez les patients en sonde à demeure. Rappelons encore la difficulté et la subtilité des signes cliniques d'infection urinaire chez ces patients, l'impossibilité de pouvoir localiser l'infection entre haut et bas appareil, l'ensemble des examens classiques de localisation n'étant pas fiable chez ces patients. Si les données de la littérature ne permettent pas de dégager des facteurs prédictifs de survenue de pyélonéphrites, il convient de prévoir dans ce contexte de grossesse, la réalisation régulière d'urocultures et l'éradication systématique de toute bactériurie asymptomatique.

Les différentes études s'accordent sur le fait que l'infection urinaire se révèle d'une particulière incidence lors de la grossesse des femmes paraplégiques. Ainsi, Baker [2], dans son étude portant sur 11 femmes, montre que 10 d'entre elles ont présenté au moins une infection urinaire, dont 3 pyélonéphrites. N. Westgren [11] souligne que l'infection urinaire reste la complication la plus fréquente avec 37 épisodes pour 49 grossesses. L. L. Cross [4] a subdivisé sa population de 22 femmes ( 33 grossesses) en 4 sous-groupes en fonction du mode mictionnel. Toutes les patientes utilisant une sonde à demeure ou porteuse d'une dérivation ont au moins un épisode infectieux, $50 \%$ des patientes aux autosondages, $17 \%$ des femmes urinant par Crédé, et $40 \%$ des femmes étiquetées miction normale.

La prévention des infections urinaires passe par une diurèse abondante $(2 \mathrm{~L} / 24 \mathrm{H})$, et l'élimination de tout résidu post mictionnel, l'absence de drainage continu. Chez les femmes urinant soit de façon réflexe, soit par poussée, il est fréquent de constater notamment lors du $3^{\circ}$ trimestre une majoration de la dysurie (augmentation du volume utérin), à l'origine de résidu pouvant conduire à la mise aux autosondages. L'idéal serait qu'avant sa grossesse, la femme paraplégique puisse bénéficier d'un bilan neuro-urologique complet, afin de dépister d'éventuelles complications, des facteurs prédictifs de risques sur le haut-appareil urinaire, et d'adapter le mode mictionnel à ces facteurs. Nous estimons qu'il est possible de poursuivre les anticholinergiques (chlorure d'oxybutinine) pendant la grossesse pour éviter des fuites réflexes trop importantes.

\section{Les escarres}

Les escarres sont la première cause de réhospitalisation chez le blessé médullaire [13]. L'incidence annuelle de cette complication a été évaluée à $23 \%$, et $85 \%$ de ces blessés présentent au moins une fois dans leur vie une escarre. Chez les femmes paraplégiques enceintes, la perte de mobilité liée à la prise de poids, la difficulté à la réalisation des transferts majorent ce risque. Les modifications morphologiques peuvent conduire à des appuis délétères et le changement transitoire du fauteuil pour un fauteuil plus large est parfois nécessaire. Ce risque impose au fil des mois des temps de repos de plus en plus importants dans la journée afin de décharger les zones d'appuis. Cette complication dans la littérature est diversement évaluée : ainsi Baker [2] fait état de 2 escarres durant la grossesse chez 11 patientes. L.L. Cross [4] fait état de complications dramatiques chez une jeune femme qui s'asseyait dans la position du lotus dans son fauteuil induisant ainsi une nécrose extensive des segments jambiers avec arthrites tibiotarsiennes traitées par amputation tibiale bilatérale! Westgren [11] rapporte quant à elle la survenue de 6 escarres chez 29 patientes. Lors de suivi de la grossesse, les patientes doivent être systématiquement examinées d'un point vue cutané, l'escarre exposant aux complications infectieuses générales et aux pertes protidiques. La constatation d'une lésion impose une consultation spécialisée par des médecins paraplégistes.

\section{Maladie thromboembolique}

Majeur, à la phase aiguë, le risque de thrombophlébite reste 
toutefois important à la phase chronique chez ces patients. Ainsi de Vivo [5] a évalué le risque de décès par embolie pulmonaire à 5 ans de la lésion comme étant 8 fois plus important que dans la population normale. Ce risque est évidemment majoré durant la grossesse, et ce d'autant plus chez les flasques ou il existe un oedème de stase important. Toutefois peu de cas ont été rapportés dans la littérature, ainsi il ne semble pas raisonnable de prévoir une prophylaxie systématique hormis lors d'antécédents thromboemboliques [2]. Des mesures telle la surélévation des membres inférieurs, le port de contention efficace, la réalisation de séances de drainage lymphatique peuvent participer efficacement à la prévention.

\section{Fonction respiratoire}

De façon générale, il existe chez les femmes enceintes une diminution de la capacité résiduelle fonctionnelle et une augmentation de la consommation d'oxygène. Chez la femme présentant une lésion thoracique haute ou cervicale, la fonction respiratoire est déjà altérée (syndrome restrictif). De plus la position allongée, la plus souvent favorable à la fonction respiratoire des patients tétraplégiques, est délétère en fin de grossesse en raison d'une obstruction des espaces aériens [1]. Chez les patients à syndrome restrictif important, des mesures régulières de la capacité vitale semblent nécessaires afin d'étayer la nécessité d'une ventilation assistée, qui s'impose lorsque la capacité vitale descend en dessous de $15 \mathrm{ml} / \mathrm{kg}$. La réalisation d'exercices respiratoires l'aide au désencombrement participent à la prévention de ces complications.

\section{Autres problèmes médicaux}

\section{a) Anémie}

De survenue commune dans la population des femmes enceintes, cette complication a été rapportée comme plus significative chez les femmes paraplégiques. Dans la série de Baker [2] Son incidence est de $63 \%$, mais elle n'apparaît pas comme majeure avec un taux moyen d'hématocrite lors de l'accouchement de $36 \%$. Exposant probablement à un risque plus important d'escarres d'une part, d'autre part la nécessité de transfusion après un accouchement étant d'autant plus important en présence d'une anémie préaccouchement, l'anémie doit être identifiée et éventuellement corrigée. Une supplémentation en fer peut être requise mais doit être donnée judicieusement en raison des problèmes intestinaux.

\section{b) Spasticité}

La spasticité est mentionnée dans quelques études et particulièrement dans l'étude de Charlifue [3] comme une plainte importante des femmes interrogées. Il convient d'être prudent quant à la prescription de benzodiazépines, l'étude de Charlifue [3] rapportant 2 cas de nouveaux-nés nécessitant une intubation.

\section{c) Problèmes intestinaux}

Peu évoqués dans la littérature, les femmes rapportent une majoration de leur constipation. Sa prise en charge fait appel à une alimentation riche en fibre, une diurèse abondante, le déclenchement mécanique du réflexe d'exonération.

\section{Le $3^{\circ}$ trimestre et prépartum}

Le risque de malformations fœtales, de morts in utero, et d'hypotrophie fotale ne semble pas plus important que dans la population générale.

Le travail prématuré se définit par la survenue de contractions utérines régulières entraînant une modification du col avant 37 semaines d'aménorrhée. La revue de la littérature depuis 1985 objective que la grossesse chez la femme blessée médullaire dépasse rarement 40 semaines d'aménorrhée. Baker dans son étude portant sur 13 grossesses chez 11 femmes objective une durée moyenne de grossesse de 37.9 semaines d'aménorrhée. L'incidence d'un accouchement prématuré a été diversement évaluée dans la littérature de $18 \%$ [11] à 27\% [9], la plupart des séries faisant état de la survenue d'un accouchement prématuré entre 35 et 37 semaines d'aménorrhée $[2,4,11]$. Ce risque apparaît donc légèrement plus élevé que dans la population générale. Ce risque associé à un risque de début de travail passé inaperçu dans les niveaux supérieurs à T10, impose une surveillance accrue à partir de la $28^{\circ}$ semaine. Celle-ci doit comprendre un examen cervical une fois par semaine, pour certains auteurs le monitorage des contractions 3 fois par semaine à domicile et dans les niveaux hauts, une hospitalisation peut-être proposée à partir de la $36^{\circ}$ semaine. En fait, il est extrêmement important d'apprendre aux femmes à palper leur utérus pour dépister les contractions. L'étude de Wanner [10] objective que la plupart des femmes de niveau supérieur à $\mathrm{T} 10$ ressentent de façon indirecte leurs contractions : sensations de ballonnements, de " gaz », de tension abdominale et pelvienne, d'augmentation de la spasticité notamment abdominale, de signes d'HRA qui au début du travail sont rythmées par les contractions utérines. Chez les femmes de niveau inférieur à $\mathrm{T} 10$, la perception des contractions permet de se dispenser d'une hospitalisation pré-terme et d'un monitorage à domicile, mais pas des examens hebdomadaires.

\section{ACCOUCHEMENT}

\section{Travail et accouchement}

Un des principaux buts est de prévenir la survenue d'accouchements inopinés, la littérature rapportant quelques cas d'accouchement en catastrophe à la maison. Fort heureusement la plupart des femmes sont capables de déceler le début du travail. Le déclenchement du travail est soumis à controverses, la survenue de phénomènes d'HRA a été rap- 
portée aussi bien lors de déclenchement spontané que lors de déclenchement induit. En l'absence de données précises, il convient de réserver ce déclenchement aux indications obstétricales.

L'équipe obstétricale doit être informée du risque d'escarres et de traumatismes chez ces patientes. Si possible, la femme doit être autorisée à accoucher dans son lit pour prévenir tout traumatisme. Les complications orthopédiques, la spasticité et les contractures peuvent gêner le passage vaginal. La difficulté d'une installation en position gynécologique peut conduire à un positionnement sur le côté. La vidange vésicale doit être assurée soit par la mise en place d'une sonde à demeure, soit par la réalisation de sondages intermittents durant l'accouchement.

La physiologie du travail ne semble pas être altérée par la lésion médullaire. Baker [2] retrouve une durée moyenne de 4,6 h sans différence significative entre lésions supérieures à $\mathrm{T} 10 \mathrm{ou}$ inférieures à $\mathrm{T} 10$, durée dans les normes habituelles. L'impression clinique de rapidité est peut-être due à une perception retardée du travail chez ces patientes. Le travail est dominé par le risque d'HRA, dont les premières manifestations sont rythmées par les contractions utérines, ce risque impose la réalisation d'une anesthésie péridurale systématique dès le début du travail chez toutes les femmes de niveau supérieur à T6.

\section{Mode d'accouchement}

Le taux de césarienne dans la littérature varie de $25 \%$ [3] à $43 \%$ [4]. Westgren [11] dans sa revue de 49 grossesses chez 29 patientes fait état de $47 \%$ de césariennes dans les niveaux supérieurs à T5 versus $26 \%$ dans les niveaux sous jacents. Son étude souligne surtout le fait que grand nombre de césariennes sont pratiquées à titre systématique, soulignant ainsi la grande méconnaissance des obstétriciens en ce qui concerne la prise en charge de ces patientes. Clairement il n'existe aucune indication neurologique de césarienne, hormis le syndrome d'HRA non jugulable par l'analgésie péridurale. La deuxième indication potentielle est l'existence d'une cavité syringomyélique post-traumatique connue, les efforts de poussée pouvant conduire à son extension. Ceci nous conduira peut-être dans l'avenir à pratiquer une IRM systématique avant toute grossesse. Les indications de césariennes doivent donc être uniquement obstétricales. Rappelons à cet égard, la nécessité d'une radiopelvimétrie chez les femmes ayant un traumatisme du bassin associé, ou lors de paraplégies congénitales ou acquises lors de l'enfance ou de l'adolescence, en raison de dysmorphismes pelviens possibles.

Les difficultés d'expulsion sont réelles lorsqu'il existe une paralysie des abdominaux. Une aide par extraction instrumentale est parfois nécessaire, $22 \%$ de forceps dans l'étude de Charlifue [3].
Dans les syndromes de la queue de cheval, afin d'éviter les efforts de poussée abdominale excessive qui risque de faire apparaître un syndrome du périnée descendant, on peut être amené à réaliser un forceps haut de principe voire une césarienne.

\section{Post-partum}

La difficulté de cicatrisation d'une éventuelle épisiotomie est fréquemment soulevée dans la littérature, certains auteurs recommandant l'utilisation de fils non résorbables pour éviter toute dehiscence. Ainsi L.L. Cross [4] fait état d'une désunion sur une série de 25 grossesses. En l'absence de série prouvant la supériorité des fils non résorbables, les sutures avec du matériel résorbable peuvent être utilisées, la prévention des désunions et des infections de la cicatrice d'épisiotomie passant par la limitation du temps de station assise, l'hygiène du périnée, la maîtrise des phénomènes d'incontinence jusqu'à cicatrisation.

Les complications du post partum sont dominées par le risque de thrombophlébite, d'infections urinaires, de rétention urinaire. Chez la plupart des patientes, la fonction vésicale retrouve son statut antérieur, mais certains cas de détérioration à long terme ont été signalés. La littérature reste muette en particulier sur les conséquences à long terme sur un périnée dénervé (paraplégie flasque, syndrome de la queue de cheval), des efforts de poussée et de la distension périnéale. Quel est le risque de syndrome du périnée descendant chez ces femmes, et de majoration d'une éventuelle incontinence d'effort?

Rappelons par ailleurs que la montée de lait se fait le plus souvent normalement et qu'il n'y a aucune contre indication à l'allaitement, hormis la prise de médicaments incompatibles.

Dans l'enquête de Charlifue [3] sur le vécu de la grossesse et de l'accouchement, les patientes signalent des problèmes physiques dans $10 \%$ des cas (intestinaux, vésicosphinctériens, spasticité, thrombophlébite, fatigue, maux de tête). $50 \%$ des femmes nécessitent une assistance supplémentaire à domicile.

\section{CONCLUSIONS}

L'issue de la grossesse chez les femmes paraplégiques est le plus souvent favorable. La condition princeps est une bonne information de la femme de sa néophysiologie, la maîtrise par les équipes obstétricales de leurs complications potentielles et un suivi conjoint avec un service spécialisé.

\section{RÉFÉRENCES}

1. BAKER E. R., CARDENAS D.D. : Pregnancy in spinal cord injured women. Arch. Phys. Med. Rehabil.,1996, 77 : 501-507.

2. BAKER E. R., CARDENAS D.D., BENEDETTI T. J. : Risks 
associated with pregnancy in spinal cord injued women. Obstet. Gyneco., 1992, 80 : 425-428.

3. CHARLIFUE S.W., GERHART K.A., MENTER R.R., WHITENECK G.G., MANLEY M.S. : Sexual issues of women with spinal cord injuries. Paraplegia, 1992, 30 : 192-199.

4. CROSS L.L., MEYTHALER J.M.,TUEL S.M.,CROSS A.L. : Pregnancy, labor and delivery post spinal injury. Paraplegia, 1992, $30: 890-892$.

5. DE VIVO M.J., BLACK K.J., STOVER S.L. : Causes of death during the first 12 years after spinal cord injury. Arch. Phys. Med. Rehabil., 1993, 74 : 248-254.

6. HEAD H., RIDDOCH G. : Autonomic bladder excessive sweating and some reflex conditions in gross injuries of spinal cord. Brain, 1917, $46: 188-263$.

7. National Institute on Disability and Rehabilitation Research Consensus Statement. The prevention and the management of urinary tract infection among people with spinal cord injury. $\mathrm{J}$. Am. Paraplegia Soc., 1992.

8. ROBERTSON D.N.S., GUTTMAN L. : The paraplegic patient in pregnancy and labour. Proc. R. Soc. Med., 1963, $56: 381-387$.

9. TURK R. , TURK M., ASSEJEV. : The female paraplegic and mother-child relations. Paraplegia, 1983, 21 : 186-191.

10. WANNER M. B., RAGETH C.J., ZACH G.A. : Pregnancy and autonomic hyperreflexia in patients with spinal cord lesions. Paraplegia, 1987, $25:$ 482-490.

11. WESTGREN N., HULTING C., LEVI R., WESTGREN M. : Pregnancy and delivery in women with a traumatic spinal cord injury in Sweden, 1980-1991. Obstet. Gynecol., 1993, 81 : 926929.

12. WESTGREN N., LEVI R. : Motherhood after traumatic spinal cord injury Paraplegia, 1994, 32 : 517-523.

13. WHITENECK G. G., CHARLIFUE S.W., FRANKEL H.L., et al: Mortality, morbidity, and psychosocial outcomes of persons spinal cord injured more than 20 years ago. Paraplegia, 1992, 30 : 617-630.

\begin{abstract}
Management of pregnancy and delivery in spinal cord injured women

J.J. LABAT, B. PERROUIN-VERBE

The overall outcomes of pregnancies and deliveries in spinal cord injured women are generally favorable. However, several antepartum complications may occur, the most frequent being urinary tract infection. There is also a risk of pressure sores and thrombophlebitis. Autonomic hyperreflexia is a serious problem and a potential danger to spinal cord injured women with neurological levels above T6, particularly during the third trimester and during labor. Autonomic hyperreflexia is generally prevented by continuous epidural anesthesia. Concerning the mode of delivery, most patients are able to deliver vaginally, but forceps are sometimes necessary. Cesarean section must only be performed for obstetric reasons and in cases of intractable autonomic hyperreflexia.
\end{abstract}

Key words : Spinal cord injury, paraplegia, procreation, conception, pregnancy, reproduction, delivery 